\title{
OPTIMIZATION OF FLOATING OFFSHORE WIND TURBINE PLATFORMS WITH A SELF-TUNING CONTROLLER
}

\author{
Frank Lemmer, Kolja Müller, Wei Yu, David Schlipf and Po Wen Cheng \\ Stuttgart Wind Energy (SWE) \\ University of Stuttgart \\ 70569 Stuttgart/Germany \\ Email: lemmer@ifb.uni-stuttgart.de
}

\begin{abstract}
The dynamic response of floating offshore wind turbines is complex and requires numerous design iterations in order to converge at a cost-efficient hull shape with reduced responses to wind and waves. In this article, a framework is presented, which allows the optimization of design parameters with respect to user-defined criteria such as load reduction and material costs. The optimization uses a simplified nonlinear model of the floating wind turbine and a self-tuning model-based controller. The results are shown for a concrete three-column semi-submersible and a 10MW wind turbine, for which a reduction of the fluctuating wind and wave loads is possible through the optimization. However, this happens at increased material costs for the platform due to voluminous heave plates or increased column spacing.
\end{abstract}

\section{INTRODUCTION}

The task of designing a substructure for floating offshore wind turbines (FOWT) is a multidisciplinary problem. In the European Horizon2020-project LIFES50+ four platform design companies collaborate with universities and research institutes on the design, upscaling and testing of the three different platform types, TLP, semi-submersible (steel \& concrete) and barge. Since the LIFES50+ public platform designs are not yet available at the time of this study, the INNWIND.EU TripleSpar concept, [1] is used as a reference, see Fig. 1. Numerical simulation models represent the complex aero-hydro-servo-elastic nature of the system. In LIFES50+ D4.4, [2] the variety of the design tools used in the consortium is presented. Hence, the early design is typically done in iterative steps and with differing level of detail for the different components of the floating platform. An essential part of this procedure is to focus on the main hull shape dimensions early and apply adjustments later in the design if important requirements are not met. The herein presented optimization framework is developed as a tool supporting the designers in the conceptual design stage. The optimization objectives of this multidisciplinary problem are reduced for this task in order to meet the range of validity of the simplified simulation models and to still allow the designer to define the constraints due to manufacturing, transport and installation requirements, which are not directly included in the presented models. This will lead to feasible optima from a point of view of the dynamic behavior. The designer's experience will still be represented through constraints of the optimizer, as well as site-dependent conditions like water depth and environmental conditions. A design practice for FOWT with three stages (conceptual design, basic design and detailed design) was developed in LIFES50+ D7.4 [3].

The optimization framework presented in this paper uses parameterized and computationally efficient numerical simulation models to represent the dynamic behavior of the system. This builds up on the reduced-order numerical models that were presented in LIFES50+ D4.1 [4] that enable the designer to quickly evaluate a given design on the base of time domain analyses. Thus, the presented models also allow an evaluation of how a considered design will behave dynamically in a given environment, next to the typically performed spreadsheet design process looking at the static integrity and stability. A more detailed, highfidelity evaluation of a large number of designs at the conceptual stage of the design is difficult to include due to the numerical effort required by state-of-the-art tools for time domain analysis.

Design optimization is a topic in engineering which has been addressed extensively in the literature: A comprehensive study on hull shape and mooring line optimization of FOWT across different platform types using a genetic algorithm was done in [5]. In that work a frequency-domain model is derived 
from the code FAST [6] with a linear representation of the hydrodynamic viscous damping but without representing the wind turbine control. The genetic algorithm is applied for single- and multi-objective optimization. The results show different, rather unconventional, designs, which might indicate that a refinement of the cost function is necessary. Another work on optimization of FOWT using a spar-type platform is presented in [7]. It includes the mooring lines and the power cable but does not include the dynamics of the wind turbine and the control. The results show that the response can be optimized by modifying the cylindrical shape of a spar. Another integrated optimization approach for spar-type FOWT using the multibody code Simpack was presented in [8] and in [9] comparing three spartype platforms with tailored blade-pitch controllers. A parametric study of a semi-submersible platform for design optimization can be found in [10] and another work specific to TLPs is part of the thesis [11]. Here, especially hydrodynamic loading of first, second and third order is considered with the combination of the controller and controller faults in extreme sea states. Another work on TLP optimization for load reduction can be found in [12]. Examples for optimization studies are presented in the thesis [13], which addressed integrated design and optimization of offshore wind turbines and in [14], where a Particle Swarm Optimizer (PSO) was applied to jacket substructures. A comparable study with a Genetic Algorithm (GA) was presented in [15]. A gradient-based optimizer was applied for offshore support structures in [16]. With this optimizer it is especially important to ensure a continuous description of the cost function. An example for a parametric design model of oil \& gas support structures subject to optimization for a reduced downtime through improved seakeeping is given in [17]. Optimization studies have also been done for the mooring lines of FOWT, see [18]. Also, an example of optimization of mechanical systems using multibody approaches from a more theoretical viewpoint can be found in the thesis [19].

Specifically new to this study is the inclusion of the wind turbine controller within the optimization in order to investigate the effect of varying aerodynamic damping through the coupled dynamics of the blade pitch and the generator torque controller during operation. It was shown in [20] and [21] that it is difficult for semi-submersibles to mitigate first-order wave loads with the wind turbine controller due to the limited control authority, or limited effect of the actuators blade pitch angle and generator torque compared to the effect of wind and wave loads. Especially the blade pitch control design is a challenge for floating wind turbines because of the low-frequency dynamics of the support structure. These introduce an inverse-response behavior due to the interaction of the fore-aft motion with the control, which results in a "negative damping" of the FOWT system. This has been described in [22] for conventional single-input-singleoutput (SISO) control and recently in [23] for an improved behavior using more control inputs than the rotor speed error. Another

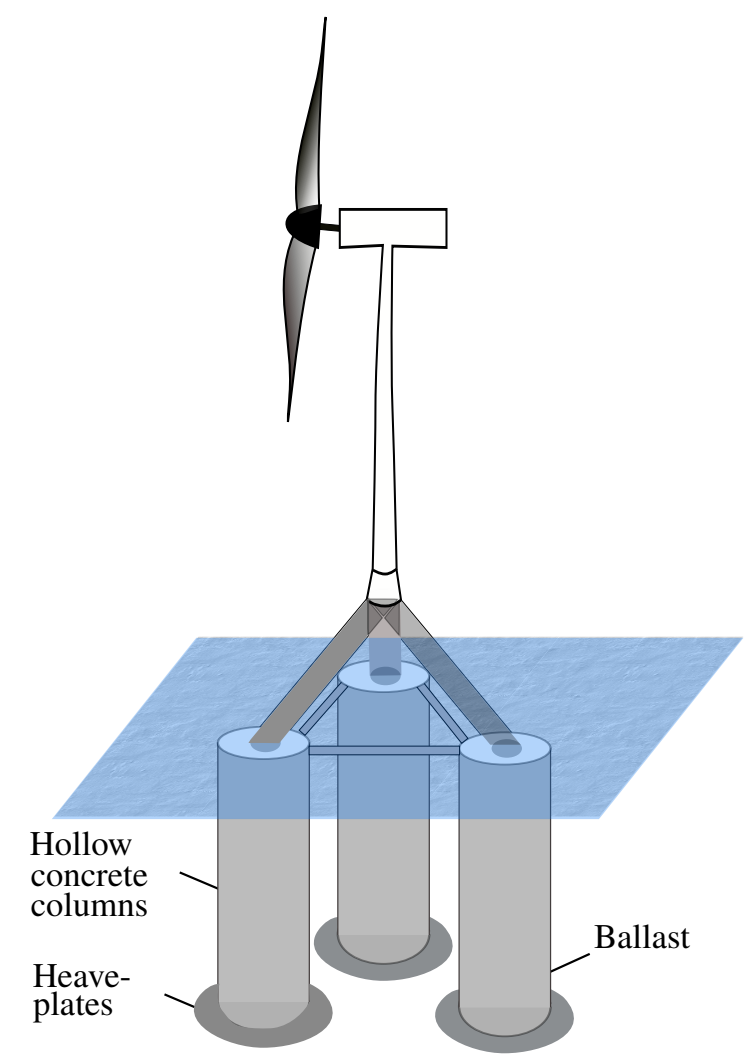

FIGURE 1. INNWIND.EU TRIPLE-SPAR CONCEPT FOR DTU10 MW WIND TURBINE (rotor diameter: $178.3 \mathrm{~m}$, hub height: $119.0 \mathrm{~m}$ ).

example of advanced controllers for FOWTs using wind preview information is [24]. In the latter study an optimal controller is applied, which is based on a linear dynamic model of the system. This model has been applied for FOWT before and was presented among others in [21], [25] and [26]. For this work the control architecture was selected such that its design depends on the actual dynamics of the current platform geometry for a parametric definition within the optimization loop. An optimal Linear Quadratic Regulator (LQR) is defined for each platform design iteration using a linear model and applying the same cost function for all platforms in order to allow a "fair" comparison between the different solutions. Consequently, a design will not be disregarded only due to a suboptimal controller. Since the LQR requires more measurements than are usually available on real turbines, the optimal controller results in the upper bound of what is possible to achieve with control. This causes the optimizer to adjust the hull shape for a further improvement of the response where the effect of the control reaches its limit.

The main objective of the optimization is to find the hull shape which experiences as little wave and wind excitation forces as possible taking into account the coupled dynamics with the controller. Here, especially the wave cancellation effect, is 
of interest, see e.g. [27]. As shown in [21] the coupled response poses a major challenge for the rotor speed control, especially at the wave frequencies. The optimization of the hull shape taking into account the controller shall indicate possible improvements in the conceptual design phase. In the design, additional input needs to be considered from a number of disciplines that play a major role in different parts of the system lifetime (e.g. engineering, production \& logistics, maintenance, economics, risk analysis, etc.). These are expected to be addressed in subsequent design stages.

The scope of the presented work is in summary:

- $\quad$ optimization variables are the geometric hull shape parameters

- $\quad$ optimization objective is the rejection of wind and wave loads at reduced material cost of the platform

- $\quad$ wind turbine controller is adjusted for each concept using an optimal, model-based control architecture

- $\quad$ design stage is conceptual, simplified simulation models are applied

- $\quad$ platform type is a three-column semi-submersible.

- $\quad$ neglected are detailed aspects of manufacturing, installation, operation and maintenance

\section{METHODOLOGY}

In order to reduce the complexity of the optimization the number of free variables was set to a minimum. This means that the designer is expected to define clear bounds of feasible concepts. The design space will be a combination of hull shape parameters, while the wind turbine is unchanged, except for the blade-pitch and generator torque control. The model used is the DTU10 MW reference wind turbine, described in LIFES50+ D1.2 [28] and [29]. In the presented framework a variety of design parameters are functions of the free variables. This means that an automated design of the components based on the free variables is done. This is called "subsystem design" and is included in the optimization algorithm, see blue boxes in Fig. 2. In particular, the preliminary structural design and the control design are done within subsystem design routines. An internal root-finding algorithm determines one of the hull shape parameters based on the constraint of the static pitch angle of the platform under the thrust force at rated wind speed as was already done in [30]. The first step for setting up the optimization problem is the determination of the chosen free variables and the bounds which allow designs that meet the static pitch angle constraint. The optimization loop feeds these into the subsystem design procedure, where the structural dimensioning based on assumed ULS stresses is made and the mooring lines are designed, keeping the same three-line system with constant line length and shape for all designs. Once the system design is established, the hydrodynamic characteristics of the platform are determined through panel code simulati- ons (red circles in Fig. 2). In a first step, the steady state of the system is determined through a nonlinear time-domain simulation for all operational wind speeds at still water. Subsequently, linear models are derived for the same wind speeds. These models are the basis for the design of an optimal model-based controller for each of the designs. The resulting system properties are then handed over to the nonlinear simulation model, which simulates the time-domain system response for defined environmental conditions. The results are then post-processed and combined in a cost function and transferred back to the optimization algorithm, which chooses the next combination of free variables. The cost function is a function of the outputs of the routines called within the optimization loop marked in dark blue in Fig. 2. The outputs of the routines marked with lighter blue are the ones that the subsequent routines need for the further design calculations and simulations.

\section{SIMULATION MODEL}

Computationally efficient simulation models are necessary for integrated optimization in order to allow for numerous iterations. The results of the subsystem designs, Fig. 2, are inputs to the SLOW (Simplified Low-Order Wind turbine) model in preparation for time-domain simulations. The model, developed at the University of Stuttgart, allows a fast simulation of the overall nonlinear coupled dynamics and is also explained in more detail in LIFES50+ D4.1 [4], [31] and [32].

The coupled FOWT system is modeled in SLOW as a multibody system of rigid or flexible bodies, which are implemented as modally reduced bodies. The equations of motion (EQM) are set up from a physical perspective following the Newton-Euler formalism. As a result, the mathematical model is available in state-space formulation as a system of symbolic ordinary differential equations (ODE), which can be directly compiled, yielding a high computational efficiency. The state vector $\mathbf{x}$, which consists of the vector of the degrees of freedom $\mathbf{q}$ and its derivative $\dot{\mathbf{q}}$ is here selected as $\mathbf{q}=\left[x_{p}, z_{p}, \beta_{p}, x_{t}, \Omega\right]^{T}$ as platform surge, heave and pitch displacement, tower-top fore-aft displacement due to deformation and the rotor speed. This is a minimal set of Degrees of Freedom (DOF), which allows obtaining a good overview of the main dynamics and as a tool for controller design in the conceptual design phase.

The aerodynamic loads are modeled as quasi-static integral forces on the rigid rotor disk. The aerodynamic coefficients for axial thrust and torque are calculated using Blade-Element Momentum theory (BEM) and stored as a function of tip-speed ratio and blade-pitch angle.

Hydrodynamic excitation forces are modeled with the panel code Ansys Aqwa and converted to the time-domain for simulation in SLOW. For the time-domain conversion of the radiation properties the convolution integral of Cummin's equation is neglected and the added mass is assumed to be independent of 


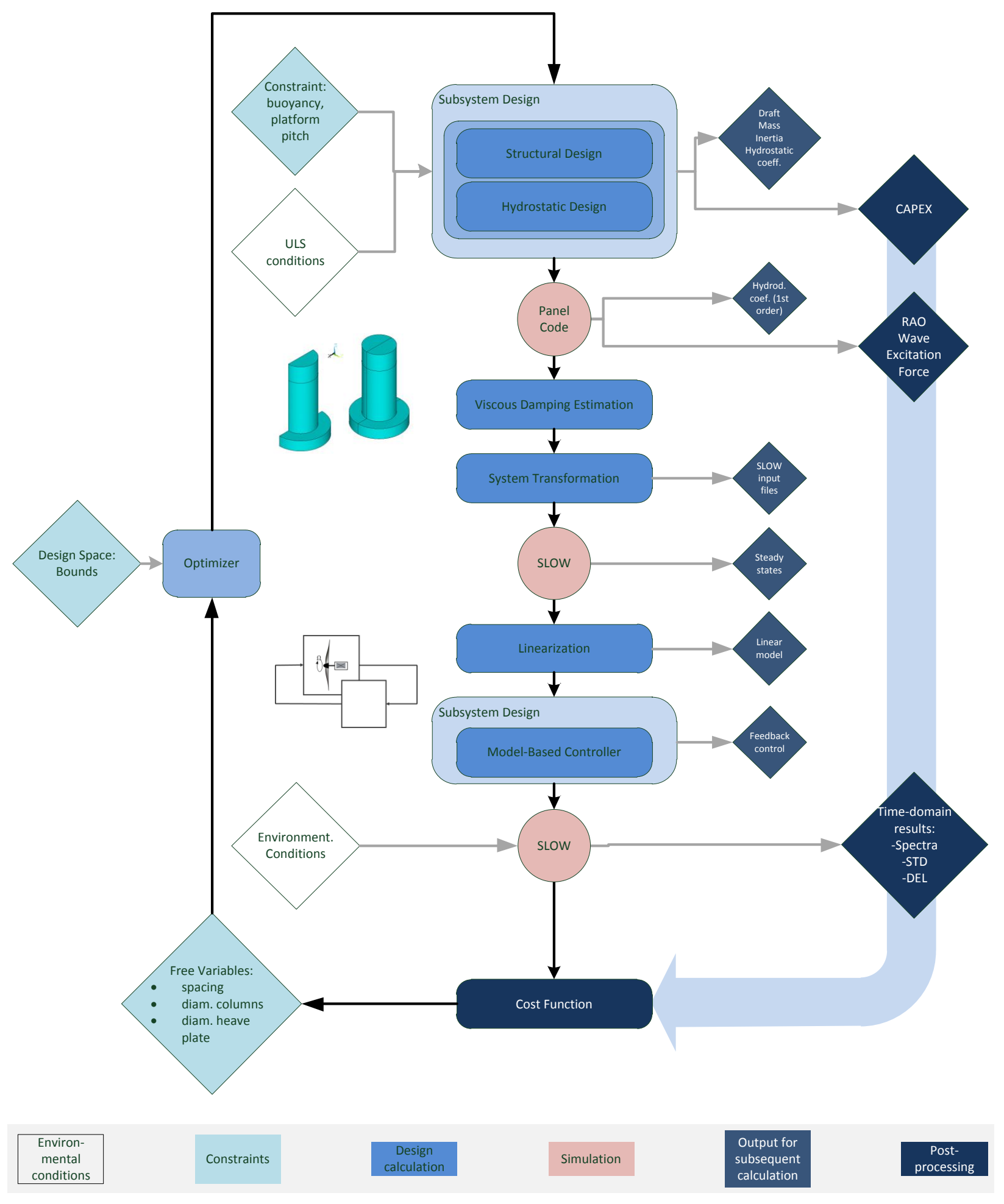

FIGURE 2. WORKFLOW OF OPTIMIZATION PROCEDURE. 


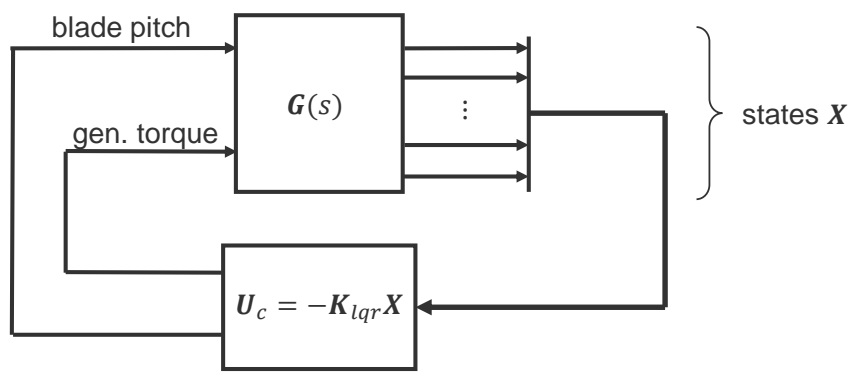

FIGURE 3. LINEAR QUADRATIC REGULATOR BLOCK DIAGRAM.

the frequency of oscillation. It is the panel code result, interpolated at the respective eigenfrequency. This method allows a significant increase of the computational speed because of the avoidance of numerical recursions. The mooring lines are quasistatic look-up tables with the nonlinear force-displacement relationships.

\section{CONTROL}

For wind speeds up to rated, a common nonlinear statefeedback law is used. This wind speed region is not critical for FOWT as, usually, it does not introduce a "negative damping" to the overall system as is the case for the blade-pitch control above rated conditions. The original controller of the DTU10 MW reference turbine, described in [29], is not used here because it is designed for a fixed foundation and includes a number of features which go beyond the standard implementation of a variable speed pitch-controlled turbine, like additional gains to increase the damping of certain modes in the closed loop. In order to keep things as simple as possible, the below-rated controller is designed comparably to the NREL5MW reference wind turbine [33]. It was adapted to the DTU10 MW reference wind turbine for LIFES50+ D4.1, [4]. A description of this controller can be also found in [1]. For wind speeds above rated an optimal Linear Quadratic Regulator (LQR) is designed, a state-feedback controller with a diagonal feedback matrix $K_{l q r}$, see Fig. 3. The two control outputs are the blade pitch angle $\theta$ and the generator torque $M_{g}$. The states of the frequency-domain SLOW model $\mathbf{X}(\omega)$, as described above, are all inputs to the controller. The feedback gain matrix $\mathbf{K}_{l q r}$ can be determined with the cost function $J$ and the weights on states $\mathbf{Q}_{c}$ and the weights $\mathbf{R}_{c}$ on the control inputs $\mathbf{u}_{c}$

$$
J=\lim _{T \rightarrow \infty} \frac{1}{T} \int_{0}^{T}\left[\mathbf{x}^{T} \mathbf{Q}_{c} \mathbf{x}+\mathbf{u}_{c}^{T} \mathbf{R}_{c} \mathbf{u}_{c}\right] d t
$$

by applying the Riccati equation, see [34]. There are no sensor models and observers included in this controller but the control inputs are assumed to be perfectly measurable. This results in an optimal feedback control, which will behave as determined by the weights $\mathbf{Q}_{c}$ and $\mathbf{R}_{c}$, independent of the floating platform geometry. Thus, the optimization algorithm will take the control dynamics into account but ensures that the comparison of the properties of the platform is not biased by the control. The performance of this controller and the ability to mitigate excitations from wind and waves was shown in [21]. The diagonal entries of the weight matrices $\mathbf{Q}_{c}$ and $\mathbf{R}_{c}$ are given as functions of the rated values (see [29]) or maximum allowable excursions. The state weights are all zero except for the azimuth angle $\varphi$, the rotor speed $\Omega$, the tower-top velocity $\dot{x}_{t}$ and the platform pitch velocity $\dot{\beta}_{p}$. The weights on the states and the control inputs generator torque $M_{g}$ and blade pitch angle $\theta$ are

$$
\begin{aligned}
& Q_{c, \varphi}=\frac{1}{\left(10.0 \Omega_{\text {rated }}\right)^{2}} \quad R_{c, M_{g}}=\frac{0.4}{M_{g, \text { rated }}^{2}} \\
& Q_{c, \Omega}=\frac{1}{\Omega_{\text {rated }}^{2}} \quad R_{c, \theta}=\frac{0.001}{\left(5.0 \frac{\pi}{180}\right)^{2}} \\
& Q_{c, \dot{x}_{t}}=\frac{0.005}{(0.22 \pi)^{2}} \\
& Q_{c, \dot{\beta}_{p}}=\frac{0.05}{(0.00142 \pi)^{2}} .
\end{aligned}
$$

\section{DESIGN ASSUMPTIONS}

Depending on the free variables, introduced in the next section, several parametric design scripts will be executed, see blue boxes in Fig. 2. The steel tripod is dimensioned based on previously found parameterizations ensuring structural integrity. Details can be found in [35]. One free hull shape variable will be determined based on the other free variables in order to satisfy the constraint of a hydrostatic restoring in pitch of $C_{55}=2.922 \times 10^{9} \mathrm{Nm} / \mathrm{rad}$ resulting in a steady-state platform pitch angle at rated wind speed of $\beta_{p, \text { rated }}=3.5 \mathrm{deg}$.

Table 1 contains the assumptions for the structural properties and the material price estimates. The concrete columns are assumed to be built with pre-stressed concrete following the example of the AFOSP spar design, see [36]. The wall thickness assumption is based on the same project and is not subject to a subsystem design loop here but constant for all geometries. The material cost is a lump cost for the processed material, meaning the total cost including manufacturing and assembly costs. The steel cost value is a contribution by LIFES50+ partner Olav Olsen and the concrete cost is an average of the value of the project AFOSP and the value from Olav Olsen due to the large difference between the values. It needs to be mentioned that these values are very rough indications, which can vary due to concrete shrinkage and also due to price variations over time.

The freeboard is not included in the structural subsystem design for reasons of simplicity. A height of the tower base of $10 \mathrm{~m}$ above Sea-Water Level (SWL) was assumed for all designs. The 
TABLE 1. STRUCTURAL DESIGN ASSUMPTIONS

\begin{tabular}{llc}
\hline Parameter & & Value \\
\hline Concrete column wall thickness & {$[\mathrm{m}]$} & 0.4 \\
Reinforced concrete average density & {$\left[\mathrm{kg} / \mathrm{m}^{3}\right]$} & 2750.0 \\
Steel density & {$\left[\mathrm{kg} / \mathrm{m}^{3}\right]$} & 7750.0 \\
Ballast density & {$\left[\mathrm{kg} / \mathrm{m}^{3}\right]$} & 2500.0 \\
Processed steel cost & {$[€ / \mathrm{kg}]$} & 4.5 \\
Processed concrete cost & {$[€ / \mathrm{kg}]$} & 0.399 \\
\hline
\end{tabular}

compliance of the final design concerning the freeboard restrictions needs to be verified in the subsequent preliminary design.

The viscous hydrodynamic damping, which is usually dominant over the potential flow damping for column-based semisubmersibles is estimated here based on the damping ratios of the SWE-TripleSpar design [1]. As a consequence the damping ratio in percentage of the critical damping is constant for all hull shapes. This means that the viscous drag depending on the column dimensions and the heave plate geometry is neglected. This is a simplification, which still ensures that the cancellation of wave excitation forces and the dynamic interaction of the FOWT system with the controller is accounted for and can be reasonably investigated with this setup.

\section{OPTIMIZATION}

Prior to the optimization, a thorough exploration of the design space is done using Design of Experiments (DoE) methods. An example of applying DoE for sensitivity analyses can be found in [37]. This is necessary as an initial study to understand the main effects of the selected free variables and, if necessary, modify the initial set of free variables and their bounds. The procedure is described in more detail in the report [35]. Subsequently, the evaluation of the results and the selection of a valid cost function are made.

\section{Design Space}

The selected hull shape parameters include the column spacing $d$ from the platform centerline, the column radius $r$, the heave plate height $h_{h p}$ and the ratio $r_{h p}$ of heave plate radius $r_{h p}$ to column radius $r$, see Fig. 4. For this work, it was decided to define upper and lower bounds for each of the free variables. This means that the design space is Cartesian, meaning that the range of every variable does not depend on the values of the other variables. This can be illustrated looking at the range of heave plate radii: For small column spacings and large column radii a design with the largest heave plate radius ratio might not be feasible. The draft, which is a result of the hydrostatic constraints, see Fig. 2, is not an actual constraint of the optimization but an upper limit of $60 \mathrm{~m}$ was considered in the a-priori definition of the bounds of the free variables.

In order to realize such a Cartesian design space a nonlinear function of the heave plate radius ratio $\hat{r}_{h p}$ was set up as a function of the column spacing $d$ and the desired heave plate radius ratio $\hat{r}_{h p, i n}$. This function ensures that the heave plates never touch each other.

\section{Cost Function}

In a first step (run \#1) the cost function to be minimized by the optimization algorithm is the Damage-Equivalent Load (DEL) of the tower-top displacement, Weibull-weighted over all wind bins. The objective here is to find the hull-shape with the least amplification of wind and wave loads on the towertop displacement. In a next step (run \#2) a cost function with weighted DEL and the Capital Expenditures (CAPEX) according to Tab. 1 was used. The normalization of both quantities uses the results of a design-space exploration run with 100 example designs. For run $\# 2$ the cost function includes a normalization and weighting of the two objectives DEL $\Delta \sigma_{e q}$ and CAPEX $c$ using the DoE-results (index $D O E$ ):

$$
J=0.6 \frac{\Delta \sigma_{e q}-\Delta \sigma_{e q, D O E, \min }}{\Delta \sigma_{e q, D O E, \max }-\Delta \sigma_{e q, D O E, \min }}+\frac{c-c_{D O E, \min }}{c_{D O E, \max }-c_{D O E, \min }} .
$$

The weighting of 0.6 results from the linear fit of the correlation between DEL and CAPEX from the DoE run.

\section{Design Load Cases}

As a basis for the Design Load Cases (DLC) for the SLOW simulations within the optimization, the "medium" site of the LIFES50+ sites was used, see [38], p.36. As mentioned previously, the focus is the behavior during operation and thus, only DLC 1.2 of IEC61400-1 is simulated. Fault conditions and extreme conditions are not covered and need to be addressed in the detailed

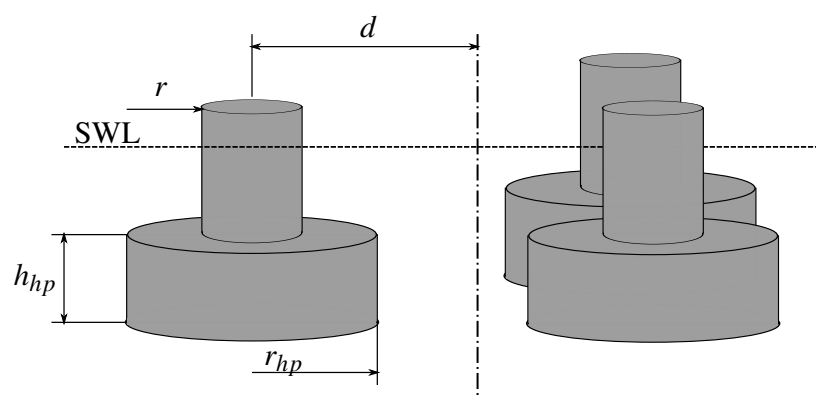

FIGURE 4. HULL SHAPE OPTIMIZATION: FREE VARIABLES. 
TABLE 2. ENVIRONMENTAL CONDITIONS.

\begin{tabular}{cccc}
\hline$v_{\text {hub }}[\mathrm{m} / \mathrm{s}]$ & $H_{S}[\mathrm{~m}]$ & $T_{p}[\mathrm{~s}]$ & $P[\%]$ \\
\hline 5.0 & 1.38 & 7.0 & 49.9 \\
7.1 & 1.67 & 8.0 & 21.6 \\
10.3 & 2.2 & 8.0 & 19.1 \\
13.9 & 3.04 & 9.5 & 7.5 \\
17.9 & 4.29 & 10.0 & 1.7 \\
22.1 & 6.2 & 12.5 & 0.2 \\
25.0 & 8.31 & 12.0 & 0.02 \\
\hline
\end{tabular}

design stage. The large excursions encountered in these DLCs exceed the range of validity of the simplified simulation models and more computationally expensive methods become necessary. In the design basis [38] three different wave environments are given, here the second one is used and therefore the probability distribution of the same source is not used but it is assumed that the second wave environment holds for each wind speed $v_{h u b}$. This results in the new probabilities according to the given Weibull distribution shown in Tab. 2. The peak spectral period $T_{p}$ for rated wind speeds, important for the evaluation of the wave excitation force coefficient is about $9 \mathrm{~s}$ and the significant wave height $H_{s}$ increases with the wind speed. The first evaluation of the system response of all designs within the bounds showed that the designs were feasible in terms of hydrostatics and hydrodynamics. However, the control design method using the LQR yields a limit-cycle behavior for the significant wave heights above $6 \mathrm{~m}$, which corresponds to the wind bins of $22.1 \mathrm{~m} / \mathrm{s}$ and $25.0 \mathrm{~m} / \mathrm{s}$, see Tab. 2. This means neglecting a total of $0.22 \%$ of operational time at the severest conditions. This issue will be addressed through further tuning of the LQR weights in future studies. It is expected that the main findings should still hold as the range of peak spectral frequencies is still wide enough to prove that the wave cancellation sought by the hull shape optimization is not an effect of a very narrow band of frequencies but holds over a range of operational conditions. Each simulation runs for one hour to ensure a good resolution of the spectra and a repeatability considering the stochastic forcing.

\section{Results}

Optimization run \#1 was done with the tower-top DEL as cost function, only and run \#2 also including the CAPEX, see eqn. 3. All other settings are equal for both runs. For both runs the Pattern Search algorithm of Matlab, described in the previous section, was used as it showed the best performance in preliminary tests using interpolations of the DoE results. It was found that the performance of the optimizer depends strongly on the selection of the initial point. Therefore, it was chosen to be as close as possible to the expected optimum (from the knowledge of the DoE, or run \#1, respectively). The settings for both runs can be found in Tab. 3. Figure 5 shows the hull shapes of the best performing design of run \#1 (DEL only). It can be seen that large column radii and large heave plates combined with a low draft lead to a significant cancellation of the wave excitation forces and thus, both results have a column radius equal to the upper limit. Looking at the CAPEX of this concept, Tab. 3, second column, it can be seen that the large heave plate radius requires a large amount of concrete, due to the quadratic influence of the radius on the mass. The benefit here is not the increased mass, but the reduced draft, which requires an increased column radius to fulfill the hydrostatic restoring requirement in pitch direction. When using the cost function of (3) for run \#2, where the CAPEX is included, the heave plates are reduced to a minimum, see Fig. 6 . The DEL is here still reduced by adjusting the other free variables. For comparison, the mass of the reference TripleSpar is $28,000 \mathrm{t}$ with a cost of $10.3 \mathrm{M} €$ and a DEL of the tower-top displacement for the same load case of 0.6, showing a clear improvement through the optimization.

In order to understand the frequency response of the resulting designs the power spectral density (PSD) is plotted for the wind speed of $v_{0}=13.9 \mathrm{~m} / \mathrm{s}$, the wave height, the towertop displacement, the rotor speed and the platform pitch angle in Fig. 7. It can be seen that the least performing design of the DoE-run (blue) shows the largest amplification of the wave

TABLE 3. OPTIMIZATION RESULTS.

\begin{tabular}{ccc}
\hline & Run \#1 & Run \#2 \\
\hline Optimizer & Pattern Search & Pattern Search \\
Cost function & $f$ (DEL) & $f$ (DEL, CAPEX) \\
Number of evaluations & 175 & 120 \\
Column radius & $10.0 \mathrm{~m}$ & $10.0 \mathrm{~m}$ \\
Column spacing & $22.14 \mathrm{~m}$ & $24.0 \mathrm{~m}$ \\
Heave plate thickness & $9.73 \mathrm{~m}$ & $1.0 \mathrm{~m}$ \\
Heave plate radius ratio & 1.7 & 1.0 \\
Draft & $24.4 \mathrm{~m}$ & $31.6 \mathrm{~m}$ \\
Platform mass & $38,000 \mathrm{t}$ & $29,000 \mathrm{t}$ \\
Result: DEL & 0.2357 & 0.3353 \\
Result: CAPEX & $12.1 \mathrm{M} €$ & $9.4 \mathrm{M} €$ \\
\hline
\end{tabular}




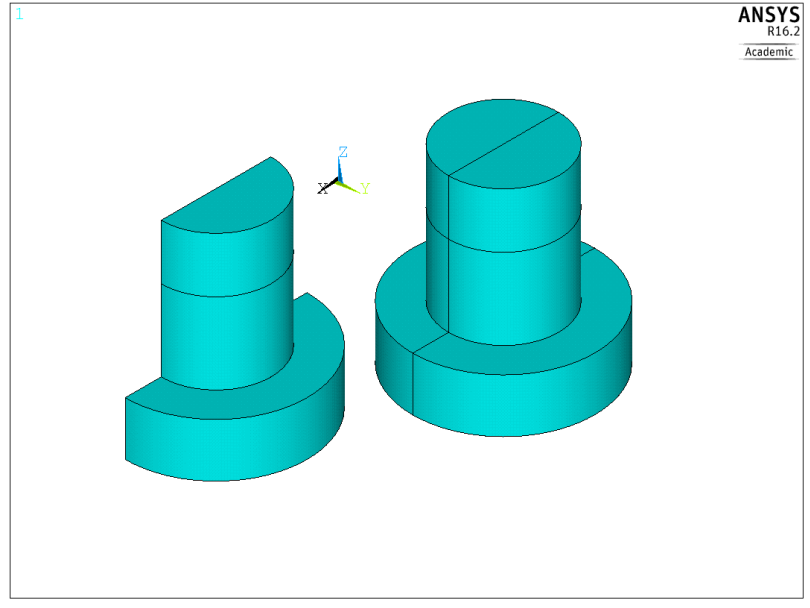

FIGURE 5. RUN \#1 OPTIMUM (SWL at horizontal line).

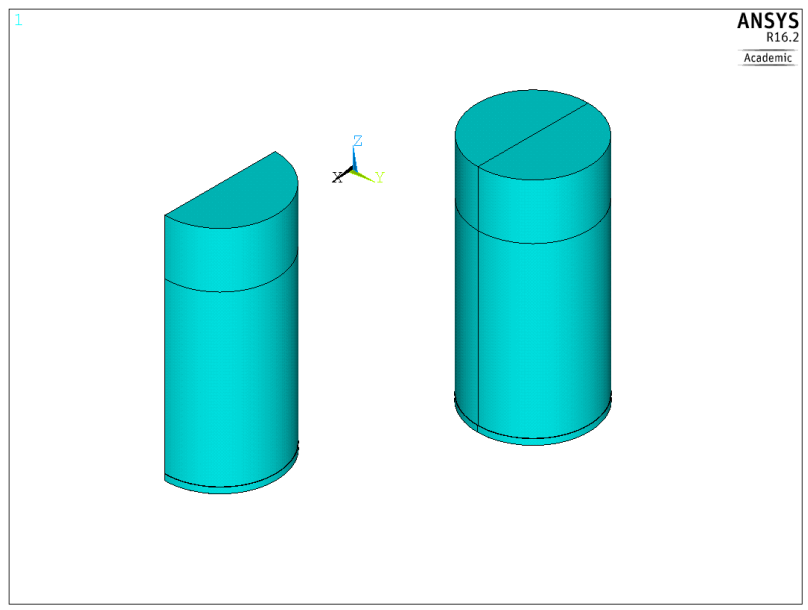

FIGURE 6. RUN \#2 OPTIMUM (SWL at horizontal line).

loads at $f_{p}=0.1 \mathrm{~Hz}$ on tower-top displacement and rotor speed. That design has a draft of $56 \mathrm{~m}$ and slender columns, it is shown in [35]. The optimum of run \#2 (DEL and CAPEX) gets less excitation at the same frequency and the optimum of run \#1 (DEL) features a notably reduced response. The response at low frequencies is also different but here the neglected slow drift excitation might yield differences for higher-fidelity simulations.

\section{CONCLUSIONS}

The article presented a framework for the optimization of FOWT systems using simplified simulation models focusing on the reduction of excitations from environmental forcing during operation. Especially new is the inclusion of the wind turbine controller into the optimization loop where a linear model is included for control design and a nonlinear model for the calcula- tion of the time domain response. It could be shown that with the given set of free variables of a concrete semi-submersible platform it is possible to significantly reduce the response amplitude for the given site. However, this happens at the cost of increased expenditures for the material due to an increased structural mass. With such a low-draft semi-submersible with thick heave plates a design for wave cancellation is possible with a significant reduction of the response of, e.g., the tower-top and the rotor speed to waves. It will be elaborated in the future how the found optima differ from the ones found through other design approaches, e.g., neglecting the controller. Other next steps include an analysis of other optimal control approaches which are not only based on the system properties but also on the disturbance model properties, e.g. $H_{\infty}$ control. In terms of model fidelity the simplified hydrodynamic damping model will be assessed in more detail in order to quantify the accuracy for the full range of platform models. For a speed increase frequency-domain calculations instead of nonlinear time-domain simulations are conceivable.

In a next step in LIFES50+ the optimization framework will be applied to optimize the two generic concepts to be selected in spring 2017. While the general findings are considered valid, a higher-fidelity study in the subsequent design phases, including other load cases and ultimate loads, is necessary to prove the results and to proceed with the detailed subsystem design (e.g. detailed structural design, mooring design, etc.).
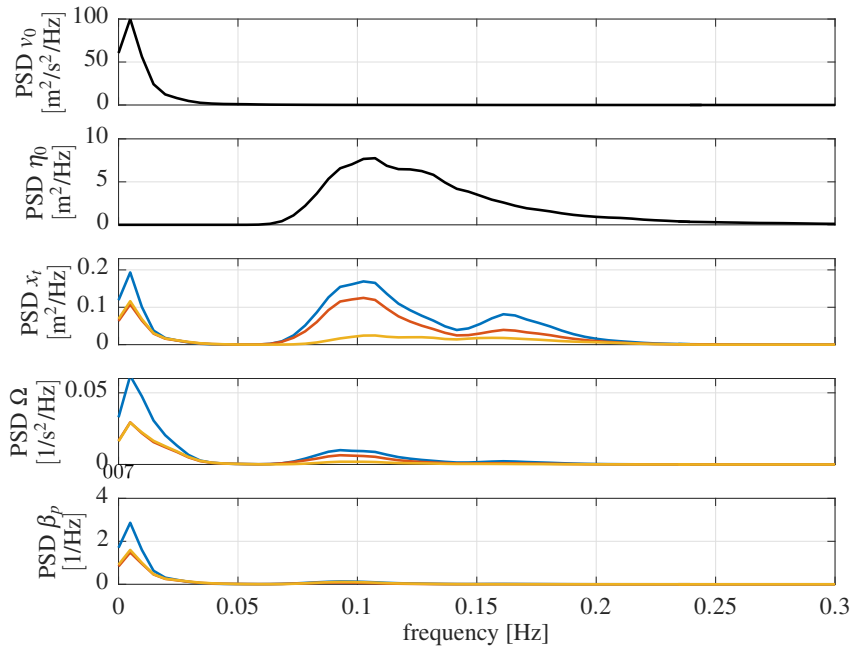

FIGURE 7. Power spectral densities of wind and wave height, tower-top disp, rotor speed and platform pitch angle; Least favorable DoE (blue), optimum cost, run \#2 (red), optimum DEL, run \#1 (yellow). 


\section{ACKNOWLEDGMENT}

The research leading to these results has received funding from the European Union's Horizon 2020 research and innovation programme under grant agreement No. 640741 (LIFES50+).

\section{REFERENCES}

[1] Lemmer, F., Amann, F., Raach, S., and Schlipf, D., 2016. Definition of the SWE-TripleSpar Floating Platform for the DTU 10MW Reference Wind Turbine. Tech. rep., University of Stuttgart.

[2] Borg, M., and Bredmose, H., 2015. LIFES50+ D4.4 Overview of the numerical models used in the consortium and their qualification. Tech. rep., DTU.

[3] Müller, K., Lemmer, F., Borisade, F., Kretschmer, M., Gruber, J., Hagemann, L., Nguyen, N.-D., and Vita, L., 2015. LIFES50+ D7.4 State-of-the-Art FOWT design practice and guidelines. Tech. rep., University of Stuttgart.

[4] Lemmer, F., Müller, K., Pegalajar-Jurado, A., Borg, M., and Bredmose, H., 2016. LIFES50+ D4.1: Simple Numerical Models for Upscaled Design. Tech. rep., University of Stuttgart.

[5] Hall, M., Buckham, B., and Crawford, C., 2013. "Evolving Offshore Wind : A Genetic Algorithm-Based Support Structure Optimization Framework for Floating Wind Turbines". In OCEANS 2013 MTS/IEEE Conference Proceedings.

[6] NWTC, 2016. NWTC Information Portal (FAST v8).

[7] Fylling, I., and Berthelsen, P. A., 2011. "WINDOPT- An optimization tool for floating support structures for deep water wind turbines". In Proceedings of ASME International Conference on Ocean, Offshore and Artic Engineering.

[8] Härer, A., Matha, D., Kucher, D., and Sandner, F., 2013. "Optimization of offshore wind turbine components in multi-body simulations for cost and load reduction". In Proceedings of the EWEA Offshore.

[9] Sandner, F., Schlipf, D., Matha, D., and Cheng, P. W., 2014. "Integrated Optimization Of Floating Wind Turbine Systems". In Proceedings of the ASME 33rd International Conference on Ocean, Offshore and Arctic Engineering.

[10] Aubault, A., Cermelli, C., and Roddier, D., 2007. "Parametric Optimization of a Semi-Submersible Platform With Heave Plates". In Volume 1: Offshore Technology; Special Symposium on Ocean Measurements and Their Influence on Design, Asme, pp. 471-478.

[11] Bachynski, E., 2014. "Design and Dynamic Analysis of Tension Leg Platform Wind Turbines". PhD thesis, NTNU.

[12] Myhr, A., and Nygaard, T. A., 2012. "Load Reductions and Optimizations on Tension-Leg-Buoy Offshore Wind Turbine Platforms". pp. 232-239.

[13] Kühn, M., 2003. "Dynamics and Design Optimization of
Offshore Wind Energy Conversion Systems". PhD thesis, TU Delft.

[14] Häfele, J., and Rolfes, R., 2016. “Approaching the ideal design of jacket substructures for offshore wind turbines with a Particle Swarm Optimization algorithm". In Proceedings of the Twenty-sixth (2016) International Ocean and Polar Engineering Conference, pp. 156-163.

[15] Schafhirt, S., Zwick, D., and Muskulus, M., 2014. "Topology Optimization of a Jacket Structure for an Offshore Wind Turbine with a Genetic Algorithm". Journal of Ocean and Wind Energy, 1, pp. 209-216.

[16] Chew, K.-h., Tai, K., and Muskulus, M., 2015. "Optimization of Offshore Wind Turbine Support Structures Using Analytical Gradient-Based Method". In EERA Deepwind.

[17] Birk, L., Clauss, G. F., and Lee, J. Y., 2004. "Practical Application Of Global Optimization To The Design Of Offshore Structures". In Proceedings of the 23rd International Conference on Offshore Mechanics and Arctic Engineering.

[18] Brommundt, M., Krause, L., Merz, K., and Muskulus, M., 2012. "Mooring System Optimization for Floating Wind Turbines using Frequency Domain Analysis". Energy Procedia, 24, pp. 289-296.

[19] Kurz, T., 2013. "Symbolic Modeling and Optimization of Elastic Multibody Systems". PhD thesis, University of Stuttgart.

[20] Fleming, P., Peiffer, A., and Schlipf, D., 2016. "Wind Turbine Controller To Mitigate Structural Loads On A Floating Wind Turbine Platform". In Proceedings of the ASME 35th International Conference on Ocean, Offshore and Arctic Engineering.

[21] Lemmer, F., Schlipf, D., and Cheng, P. W., 2016. "Control design methods for floating wind turbines for optimal disturbance rejection". Journal of Physics: Conference Series, 753.

[22] Jonkman, J., 2008. "Influence of Control on the Pitch Damping of a Floating Wind Turbine”. In Proceedings of the ASME Wind Energy Symposium.

[23] Fischer, B., and Loepelmann, P., 2016. "Balancing rotor speed regulation and drive train loads of floating wind turbines". Journal of Physics: Conference Series, 753.

[24] Schlipf, D., Simley, E., Lemmer, F., Pao, L., and Cheng, P. W., 2015. "Collective Pitch Feedforward Control of Floating Wind Turbines Using Lidar". Journal of Ocean and Wind Energy, 2(4), pp. 223-230.

[25] Raach, S., Schlipf, D., Sandner, F., Matha, D., and Cheng, P. W., 2014. "Nonlinear Model Predictive Control of Floating Wind Turbines with Individual Pitch Control". In Proceedings of the American Control Conference.

[26] Lindeberg, E., 2009. "Optimal Control of Floating Offshore Wind Turbines". MSc thesis, Norwegian University of Science and Technology. 
[27] Hanna, S. Y., 1986. "Wave Cancellation Effects and Extreme Wave Dynamics". In Offshore Technology Conference.

[28] Borg, M., and Bredmose, H., 2015. LIFES50+ D1.2 Wind turbine models for the design. Tech. rep., DTU.

[29] Bak, C., Zahle, F., Bitsche, R., Kim, T., Yde, A., Henriksen, L., Natarajan, A., and Hansen, M., 2013. Description of the DTU 10 MW Reference Wind Turbine. Tech. rep., DTU Wind Energy.

[30] Sandner, F., Yu, W., Matha, D., Azcona, J., Munduate, X., Grela, E., Voutsinas, S., and Natarajan, A., 2014. INNWIND.EU D4.33: Innovative Concepts for Floating Structures. Tech. rep., University of Stuttgart (SWE), Stuttgart.

[31] Sandner, F., Schlipf, D., Matha, D., Seifried, R., and Cheng, P. W., 2012. "Reduced Nonlinear Model of a Spar-Mounted Floating Wind Turbine". In Proceedings of the German Wind Energy Conference DEWEK.

[32] Lemmer, F., Raach, S., Schlipf, D., and Cheng, P. W., 2016. "Parametric Wave Excitation Model for Floating Wind Turbines". Energy Procedia.

[33] Jonkman, J., Butterfield, S., Musial, W., and Scott, G., 2009. Definition of a 5-MW Reference Wind Turbine for Offshore System Development. Tech. Rep. February, NREL, Boulder/USA.

[34] Skogestad, S., and Postlethwaite, I., 2007. Multivariable feedback control: Analysis and design. John Wiley and Sons, Chichester.

[35] Lemmer, F., Müller, K., Yu, W., Faerron-Guzman, R., and Kretschmer, M., 2016. LIFES50+ D4.3: Optimization Framework and Methodology for Optimized Floater Design. Tech. rep., University of Stuttgart, SWE.

[36] Molins, C., Campos, A., Sandner, F., and Matha, D., 2014. "Monolithic Concrete Off-Shore Floating Structure For Wind Turbines". In Proceedings of the EWEA.

[37] Müller, K., and Cheng, P. W., 2016. "Validation of Uncertainty in IEC Damage Calculations Based on Measurements from Alpha Ventus". Energy Procedia, 94, pp. 133145.

[38] Gómez Alonso, P., Sánchez, G., Llana, A., and Gonzales, G., 2015. LIFES50+ D1.1 Oceanographic and meteorological conditions for the design. Tech. rep., Iberdrola. 